\title{
An update on carnosine and anserine research
}

\author{
Wim Derave ${ }^{1}$ (D) Barbora De Courten ${ }^{2} \cdot$ Shahid P. Baba $^{3}$
}

Received: 10 October 2018 / Accepted: 13 December 2018 / Published online: 7 January 2019

(c) Springer-Verlag GmbH Austria, part of Springer Nature 2019, corrected publication 2019

Carnosine belongs to a family of histidyl dipeptides, consisting of L-histidine and beta-alanine. It is more prevalent in the mammalian and human tissues, whereas its analogues such as anserine, which is methylated at the $\mathrm{N}_{\pi}$ of imidazole, is present in birds and fish and balenine, methylated at the $\mathrm{N}_{\tau}$ of histidine, is largely present in marine mammals and reptiles (Boldyrev et al. 2013). Despite their early discovery and their prominent concentrations in certain tissues, the exact role and potential of these dipeptides are still not fully understood. Carnosine is a multifunctional dipeptide. Based on its biochemical properties, it has been suggested to be involved in acid-base balance, antioxidant defense and scavenging of lipid peroxidation products. The latter functions of the dipeptide have generated various expectations as therapeutic interventions, e.g. for the pathologies associated with enhanced generation of lipid peroxidation products.

There is a growing interest from the broad scientific community to unravel the fundamental properties as well as the potential application spectrum of carnosine and related dipeptides. As to date (December 2018), over 3300 publications are found on Web of Science when searching for 'carnosine', and Fig. 1 displays the fundamental (panel A) and applied research areas (panel B) in which these papers are mostly situated. The majority of fundamental research

Handling Editor: E. Closs.

This is the introduction paper to the Special Issue of Amino Acids on 'Carnosine', edited by Wim Derave, Barbora De Courten and Shahid Baba.

Wim Derave

wim.derave@ugent.be

1 Department of Movement and Sports Sciences, Ghent University, Watersportlaan 2, 9000 Ghent, Belgium

2 Monash Centre for Health Research and Implementation, School of Public Health and Preventive Medicine, Monash University, Melbourne, Australia

3 Department of Medicine, Envirome Institute, Diabetes and Obesity Center, University of Louisville, Louisville, KY, USA is performed in the field of biochemistry/molecular biology, pharmacology and physiology (Fig. 1a). The major application fields are neurology, diabetes, cardiovascular disease and nutrition (Fig. 1b), although the interest and relevance are broad and extends all the way to agriculture, zoology, sports sciences, etc. The wide variety of disciplines of the manuscripts in this current Special Issue is a reflection hereof.

In the field of diabetes, the interest in carnosine as a therapeutic target for obesity, types 1 and 2 diabetes and its complications was stimulated by the discovery of the carnosinase gene as a risk factor for diabetic nephropathy (Janssen et al. 2005). The present issue contains two valuable original contributions that follow-up on this finding. Rodriguez-Niño et al. (2019) explore the relevance of urinary carnosinase excretion in relation to renal function in type 2 diabetics, while Baye et al. (2019) expand their findings on the first carnosine supplementation trials in obese individuals (De Courten et al. 2016). A major hurdle in carnosine supplementation studies is the low stability and bioavailability of carnosine due to high presence of serum carnosinase in humans. Two manuscripts of this special issue propose to deal with this pharmacological challenge: Qiu et al. (2019) develop and identify a novel selective carnosinase inhibitor, carnostatine. On the other hand, Everaert et al. (2019) explore the pharmacokinetics of an alternative approach by supplementing anserine, a natural analogue of carnosine, which is somewhat more resistant to hydrolysis by carnosinase.

Although the potential therapeutic applications of carnosine are broad, current research is intensified on pathologies relating to tissues with a natural abundance of the histidine-containing dipeptides, such as the myocardium. In this special issue, two novel pathophysiological mechanisms of carnosine in cardiac myocytes are uncovered by Nelson et al. (2019) and Zhao et al. (2019). Nelson et al. (2019) characterize a novel detoxification pathway mediated by carnosine, which forms conjugates with catecholaldehydes, which are pathologically reactive catecholamines metabolites generated in the myocardium. Similarly, Zhao 
Fig. 1 Number of published scientific papers, retrieved by a Web of Science search on 'carnosine' (topic), divided by general scientific discipline (a) and by applied scientific discipline (b) Source: Clarivate analytics (analysis on September 19th, 2018)

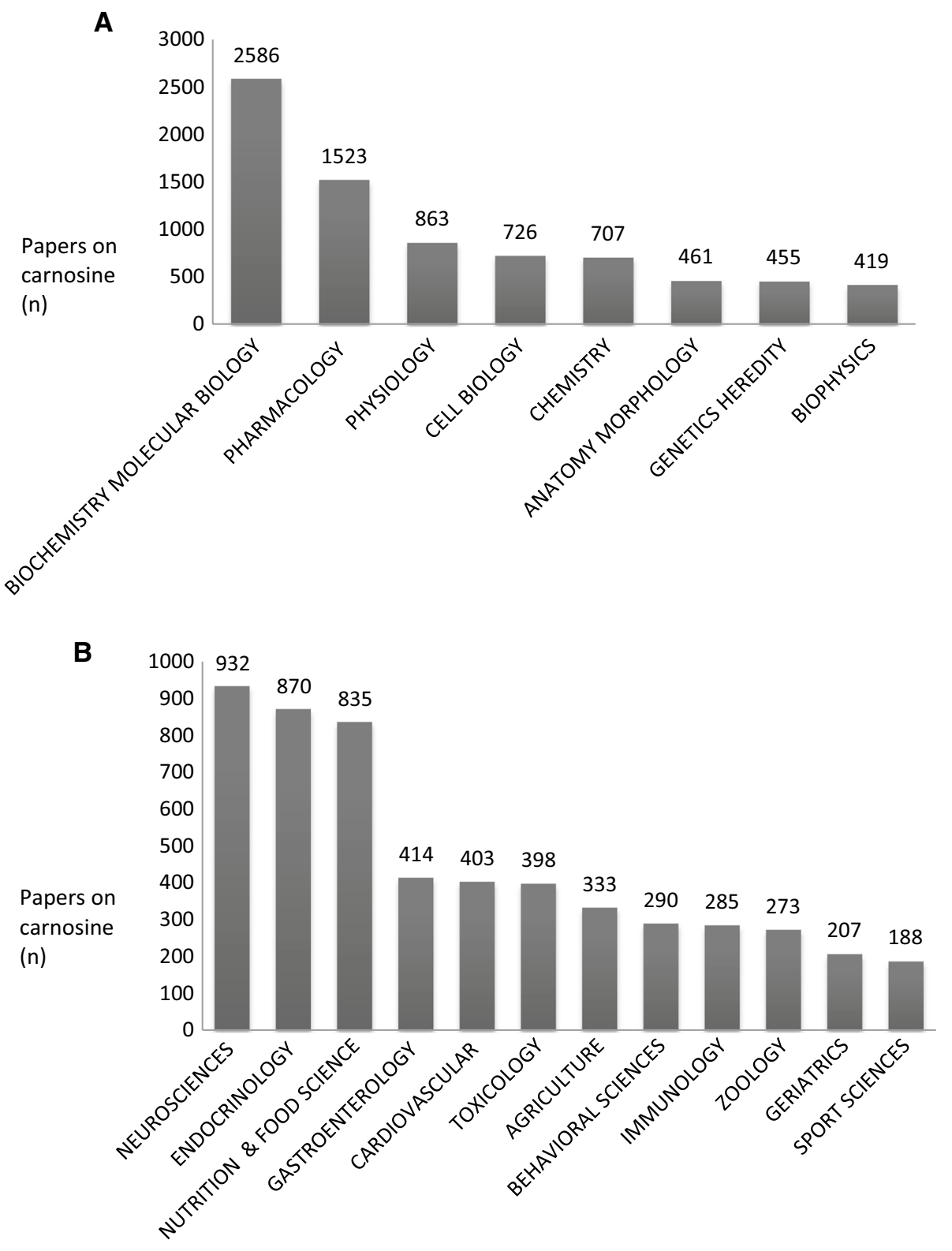

et al. (2019) provide direct evidence how carnosine could protect the adult cardiomyocytes from toxic lipid peroxidation products, generated during ischemia-reperfusion injury and heart failure. The pleiotropic action of carnosine implies therapeutic potential in various diseases, including cancer and neurodegeneration. The contribution by Oppermann et al. (2019) describes a novel level of complexity by suggesting carnosine's influence on epigenetic regulation in glioblastoma cells. Berezhnoy et al. (2019) review the role of carnosine as a neuromodulator and neuroprotector.

Apart from the various ongoing therapeutic research lines, there remains a vast interest in the normal physiological role in carnosine-rich tissues, such as skeletal muscle. Spelnikov and Harris (2019) use published experimental data to develop a kinetic model to describe carnosine synthesis in human skeletal muscle. Yet, one of the most surprising findings published in this special issue comes from Nagai et al. (2019) showing that carnosine in skeletal muscle sensitizes afferent autonomic nerves from skeletal muscles and thus for the first time suggest that the large intracellular carnosine pool in skeletal muscle cells may also serve extracellular signaling functions.

Although the role of carnosine abundance in skeletal muscle has attracted some research interest over the past century, the attention was intensified in the last decade after the discovery by Harris et al. (2006) showing that chronic dietary intake of beta-alanine can increase human muscle carnosine concentration and thereby improve exercise capacity. Three papers in this issue expand on this observation. Bassinello et al. (2019) compare three modes of muscle 
contraction (isometric, isotonic and isokinetic) to better characterize the strength potentiation of carnosine loading. Da Silva et al. (2019) elaborate on the energy delivery systems during exercise that are affected by beta-alanine supplementation. Finally, the optimal dietary mode of the actual beta-supplementation regimen is investigated by Varanoske et al. (2019).

The current special issue contains 12 valuable original contributions and 1 review paper, delivering exciting new insights and representing considerable research progress. However, they also identify a significant remaining gap in our understanding and application of carnosine and related dipeptides. Therefore, we hope that this initiative will inspire and attract more researchers to help unravel the enigmatic role of carnosine and related dipeptides; a journey that started nearly 120 years ago, with the discovery of the dipeptide in 1900 by Gulewitch (Boldyrev et al. 2013).

The special issue was composed on the occasion of and written by attendants of the fifth International Congress on Carnosine and Anserine (ICCA) which was held from 12th to 14th of September 2017, at the Diabetes and Obesity Center, University of Louisville (Dr. Shahid P. Baba and colleagues). It is endorsed by the Carnosine Consortium (http://users.unimi.it/carnosine_co/), after previous successful meetings in Moscow (2000), Ghent (2011), Tokyo (2014) and Gabicce Mare (2015). Approximately 80 researchers from all the continents gathered for an exciting 3-day conference, dedicated to the topic of carnosine and related dipeptides. The Carnosine Consortium is a common forum for the basic understanding and applications of histidyl dipeptides. This conference formally helps coalesce both established and young investigators working on disparate aspects to interact and develop a coherent view in understanding these dipeptides. A similar special issue in this journal was previously published in 2012 following the Ghent meeting (Derave and Sale 2012).

Acknowledgements We would like to thank Natural Alternatives International, the Diabetes and Obesity Center and Brand's Suntory for their financial support to the organization of the International Congress on Carnosine and Anserine.

\section{Compliance with ethical standards}

Conflict of interest We have acknowledged the financial support provided for the organization of the Conference.

\section{References}

Bassinello D, de Salles Painelli V, Dolan E et al (2019) Beta-alanine supplementation improves isometric, but not isotonic or isokinetic strength endurance in recreationally strength-trained young men. Amino Acids. https://doi.org/10.1007/s0072 6-018-2593-8

Baye E, Ukropec J, de Courten MPJ et al (2019) Carnosine supplementation reduces plasma soluble transferrin receptor in healthy overweight or obese individuals: a pilot randomised trial. Amino Acids. https://doi.org/10.1007/s00726-018-2623-6

Berezhnoy DS, Stvolinsky SL, Lopachev AV et al (2019) Carnosine as an effective neuroprotector in brain pathology and potential neuromodulator in normal conditions. Amino Acids. https://doi. org/10.1007/s00726-018-2667-7

Boldyrev AA, Aldini G, Derave W (2013) Physiology and pathophysiology of carnosine. Physiol Rev 93:1803-1845. https:// doi.org/10.1152/physrev.00039.2012

da Silva RP, de Oliveira LF, Saunders B et al (2019) Effects of $\beta$-alanine and sodium bicarbonate supplementation on the estimated energy system contribution during high-intensity intermittent exercise. Amino Acids. https://doi.org/10.1007/s0072 6-018-2643-2

De Courten B, Jakubova M, De Courten MPJ et al (2016) Effects of carnosine supplementation on glucose metabolism: pilot clinical trial. Obesity 24:1027-1034. https://doi.org/10.1002/oby.21434

Derave W, Sale C (2012) Carnosine in exercise and disease: introduction to the international congress held at Ghent University, Belgium, July 2011. Amino Acids 43:1-4. https://doi.org/10.1007/ s00726-012-1281-3

Everaert I, Baron G, Barbaresi S et al (2019) Development and validation of a sensitive LC-MS/MS assay for the quantification of anserine in human plasma and urine and its application to pharmacokinetic study. Amino Acids. https://doi.org/10.1007/ s00726-018-2663-y

Harris RC, Tallon MJ, Dunnett M et al (2006) The absorption of orally supplied beta-alanine and its effect on muscle carnosine synthesis in human vastus lateralis. Amino Acids 30:279-289

Janssen B, Hohenadel D, Brinkkoetter P et al (2005) Carnosine as a protective factor in diabetic nephropathy: association with a leucine repeat of the carnosinase gene CNDP1. Diabetes 54(8):2320-2327

Nagai K, Misonou Y, Fujisaki Y et al (2019) Topical application of 1-carnosine to skeletal muscle excites the sympathetic nerve innervating the contralateral skeletal muscle in rats. Amino Acids 6:9. https://doi.org/10.1007/s00726-018-2597-4

Nelson M-AM, Builta Z, Monroe B et al (2019) Biochemical characterization of the catecholaldehyde reactivity of 1-carnosine and its therapeutic potential in human myocardium. Amino Acids. https://doi.org/10.1007/s00726-018-2647-y

Oppermann H, Alvanos A, Seidel C et al (2019) Carnosine influences transcription via epigenetic regulation as demonstrated by enhanced histone acetylation of the pyruvate dehydrogenase kinase 4 promoter in glioblastoma cells. Amino Acids 5:6. https ://doi.org/10.1007/s00726-018-2619-2

Qiu J, Hauske SJ, Zhang S et al (2019) Identification and characterisation of carnostatine (SAN9812), a potent and selective carnosinase (CN1) inhibitor with in vivo activity. Amino Acids. https://doi.org/10.1007/s00726-018-2601-z

Rodriguez-Niño A, Gant CM, Braun JD et al (2019) Detection of carnosinase-1 in urine of healthy individuals and patients with type 2 diabetes: correlation with albuminuria and renal function. Amino Acids. https://doi.org/10.1007/s00726-018-2602-y

Spelnikov D, Harris RC (2019) A kinetic model of carnosine synthesis in human skeletal muscle. Amino Acids. https://doi. org/10.1007/s00726-018-2646-z

Varanoske AN, Hoffman JR, Church DD et al (2019) Comparison of sustained-release and rapid-release $\beta$-alanine formulations on changes in skeletal muscle carnosine and histidine content and 
isometric performance following a muscle-damaging protocol. Amino Acids. https://doi.org/10.1007/s00726-018-2609-4

Zhao J, Posa DK, Kumar V, Hoetker D, Ganesan S, Rigss DW, Bhatnagar A, Wempe MF, Baba SP (2019) Carnosine protects cardiac myocytes against lipid peroxidation products. Amino Acids. https://doi.org/10.1007/s00726-018-2676-6
Publisher's Note Springer Nature remains neutral with regard to jurisdictional claims in published maps and institutional affiliations. 